\title{
Hepatocellular carcinoma surveillance in the 21st century: Saving lives or causing harm?
}

\author{
Ibrahim A. Hanouneh', Naim Alkhouri ${ }^{2}$, and Amit G. Singal ${ }^{3}$ \\ Mayo Clinic and Minnesota Gastroenterology, Minneapolis, MN; ${ }^{2}$ Texas Liver Institute, University of Texas Health San Antonio, San \\ Antonio, TX; ${ }^{3}$ Division of Digestive and Liver Diseases, Department of Internal Medicine, UT Southwestern Medical Center, Dallas, TX, \\ USA
}

Hepatocellular carcinoma $(\mathrm{HCC})$ is the third most common cause of cancer related death worldwide. Prognosis and treatment options largely depend on tumor stage at diagnosis, with curative treatments only available if detected at an early stage. However, two thirds of patients with HCC are diagnosed at a late stage and not eligible for cure. Therefore several liver professional societies recommend HCC surveillance using abdominal ultrasound with or without alpha fetoprotein in at-risk populations, including patients with cirrhosis and subsets of those with chronic hepatitis B. Available data suggest HCC surveillance can significantly improve early tumor detection, curative treatment eligibility, and overall survival. However, the potential benefits of HCC surveillance must be considered in light a shifting HCC demographic from a viral-mediated cancer to an increasing proportion of patients having non-alcoholic steatohepatitis, which has been shown to limit ultrasound sensitivity and may mitigate observed benefits. Further, benefits of HCC surveillance must be weighed against potential physical, financial and psychological harms. Continued data for both benefits and harms of HCC surveillance in contemporary populations are necessary. In the interim, providers should continue to strive for high quality HCC surveillance in at-risk patients. (Clin Mol Hepatol 2019;25:264-269)

Keywords: Hepatocellular carcinoma; Liver cancer; Screening; Surveillance; Ultrasound

\section{INTRODUCTION}

Hepatocellular carcinoma (HCC) is the leading cause of cancer burden globally. HCC is responsible for more than 600,000 deaths per year worldwide, making it the fifth-most common cancer and the second most common cause of cancer-related mortality worldwide.' Vaccination programs against hepatitis B virus (HBV) have resulted in a decrease in HCC incidence particularly in lessdeveloped regions of the world where HBV is more prevalent. ${ }^{2}$ On the other hand, HCC is the fastest-rising cause of cancer-related death in the United States and most of Europe ${ }^{3}$ and $\mathrm{HCC}$ incidence is predicted to continue rising until $2030 .^{4}$

$\mathrm{HCC}$ is a condition that almost universally arises in individuals with underlying liver disease, hence lends itself to surveillance. At-risk individuals can be identified by the presence of chronic liver disease and surveillance can theoretically be applied in a costeffective manner to this population. Indeed, up to $90 \%$ of cases occur in patients with chronic liver disease, with most having un-

\footnotetext{
Abbreviations:

AASLD, American Association for the Study of Liver Disease; AFP, alphafetoprotein; AFP-L3, lectin-bound AFP; APASL, Asian Pacific Association for the Study of Liver; Cl, confidence interval; CT, computed tomography; DCP, desgamma carboxy prothrombin; EASL, the European Association for the Study of Liver; HBV, hepatitis B virus; HCC, hepatocellular carcinoma; MELD, model for end-stage liver disease; MRI, magnetic resonance imaging; OR, odds ratio; SEER, Surveillance Epidemiology End Result; VA, Veterans Affairs
}

\section{Corresponding author: Amit G. Singal}

Division of Digestive and Liver Diseases, Department of Internal Medicine, UT Southwestern Medical Center, 5959 Harry Hines Blvd, POB 1, Suite 420, Dallas, TX 75390-8887, USA

Tel: +1-214-645-6111, Fax: +1-214-645-6114

E-mail: amit.singal@utsouthwestern.edu

https://orcid.org/0000-0002-1172-3971 
derlying cirrhosis at time of diagnosis, ${ }^{5}$ and patients with cirrhosis have an annual HCC incidence ranging from $1 \%$ to $8 \%{ }^{5,6}$

Although the overall 1-year survival for HCC is disappointingly low, HCC can be cured if detected at an early stage via surgical resection, orthotopic liver transplantation, or ablative procedures such as microwave or radiofrequency ablation. However, more than $70 \%$ of patients diagnosed with HCC have advanced disease that is not amenable to curative therapy, in which median survival is typically between 1-3 years. The strong association between early detection and improved survival has been the impetus behind professional society guidelines for HCC surveillance among at-risk individuals, including those with cirrhosis. ${ }^{7-9}$ The American Association for the Study of Liver Disease (AASLD), the European Association for the Study of Liver (EASL) and Asian Pacific Association for the Study of Liver (APASL) recommend HCC surveillance for patients with cirrhosis with liver ultrasound with or without serum alpha-fetoprotein (AFP) test every 6 months, ${ }_{1}^{7-9}$ although other professional societies do not endorse these recommendations given concerns about the level of supporting evidence (Table 1). ${ }^{10}$ Herein, we discuss the pros and cons of HCC surveillance and its overall value among at-risk patients.

\section{IS IT POTENTIALLY BENEFICIAL?}

HCC surveillance could identify patients with early-stage tumors who may benefit from therapeutic options with potentially good long-term outcome. However, it remains unclear whether HCC surveillance actually improves overall survival in patients with liver cirrhosis or just represents early-stage migration and increased use of treatments, a phenomenon well described in other malignancies known as "over-diagnosis"."

A large randomized controlled trial of HCC surveillance demonstrated HCC surveillance can decrease cancer-related mortality in patients with chronic liver disease. ${ }^{12}$ In a large randomized con- trolled trial with over 19,000 HBV-infected individuals, those randomized to HCC surveillance were significantly more likely to be detected at an early HCC stage and more likely to undergo curative treatment. Further, HCC surveillance reduced HCC mortality by $37 \%$ (mortality rate ratio $0.63,95 \%$ confidence interval [CI] 0.41-0.98). However, this study was conducted among HBV-infected individuals in Asia and it is unclear if these results are applicable in the Western World where HCC typically arises in the background of cirrhosis.

Although there is not a similar randomized trial among patients with cirrhosis, many retrospective cohort studies have evaluated long-term outcomes in HCC patients detected by surveillance compared to those who were detected incidentally or symptomatically. ${ }^{13}$ A meta-analysis of 47 studies including a total of 15,158 found that HCC surveillance was associated with improved early detection (odds ratio [OR] 2.08, 95\% Cl 1.80-2.37), curative treatment rates (OR 2.24, 95\% Cl 1.99-2.52), and 3-year survival (OR 1.90, 95\% Cl 1.67-2.17). ${ }^{14}$ However, most studies were limited by the inherent limitations of retrospective observational studies including selection bias, and lead-time and length-time biases. A recent analysis of the Surveillance Epidemiology End Result (SEER)-Medicare database found that HCC surveillance continued to be associated with significantly higher 3-year survival after adjusting for both lead time and length time biases (23\% vs. $13 \%)^{15}$ In contrast, a case-control study in the Veterans Affairs (VA) health care system ${ }^{16}$ among 238 patients with cirrhosis who died of HCC and matched cirrhosis patients who did not die of HCC found surveillance was not associated with a decrease in cancerrelated mortality. However, it is unclear if these results reflect inherent limitations in the efficacy of surveillance or its effectiveness, i.e., how it was implemented in clinical practice. In this study, most patients only received intermittent surveillance instead of semi-annual surveillance, and this has been shown to reduce surveillance effectiveness. Further, over half of the surveillance group was detected at an early stage; however, many failed

Table 1. Practice guidelines for hepatocellular carcinoma surveillance

\begin{tabular}{lcl}
\hline & Year of publication & \multicolumn{1}{c}{ Recommendations } \\
\hline AASLD & 2018 & Ultrasound with or without AFP every 6 months \\
\hline EASL & 2018 & Ultrasound every 6 months \\
\hline APASL & 2017 & Ultrasound and AFP every 6 months \\
LAASL & 2014 & Ultrasound or AFP (if ultrasound not available) every 6 months \\
NCCN & 2018 & Ultrasound with or without AFP every 6 months \\
\hline
\end{tabular}

AASLD, American Association for the Study of Liver Diseases; EASL, European Association for the Study of Liver; APASL, Asian Pacific Association for the Study of the Liver; LAASL, Latin American Association for the Study of the Liver; NCCN, National Comprehensive Cancer Network; AFP, alpha-fetoprotein. 
to undergo curative treatment. Overall, current data are imperfect, with limitations as described above, but most studies suggest HCC surveillance has the potential to improve early detection and overall survival.

\section{IS IT POTENTIALLY HARMFUL?}

Screening-related harms can include direct complications of screening tests as well as diagnostic testing. Therefore, although ultrasound and AFP have minimal direct harms, it is important to consider false positives or indeterminate results that can lead to diagnostic testing harms. Computed tomography (CT) and magnetic resonance imaging (MRI) are associated with known harms such as contrast injury, radiation exposure, and cost as well as evolving data about harms such as accumulation of gadolinium in the brain. Further some false positive results may prompt liver biopsy, which has risk of bleeding, tumor seeding, and injury to adjacent organs. Although the literature on surveillance-related harms is more immature than data evaluating benefits, there have been a couple recent studies. Atiq and colleagues found $27.5 \%$ of patients experienced surveillance-related physical harms over a 3 -year period in a retrospective cohort study of 680 cirrhosis patients. ${ }^{17}$ Similarly, another study among 999 patients undergoing surveillance found nearly $20 \%$ of patients had an indeterminate nodule requiring diagnostic evaluation. ${ }^{18}$ Nearly three-fourths of these patients returned to ultrasound-based surveillance after a median of 2 CT/MRI studies; however, 20\% continued CT/MRI surveillance and $1 \%$ underwent invasive testing such as liver biopsy. Although these data suggest possible physical harms related to HCC surveillance, further data are needed to evaluate financial and psychological harms.

\section{IS IT COST-EFFECTIVE?}

Surveillance is considered cost-effective if can be done at a cost of less than $\$ 50,000$ for quality-adjusted life-year gained..$^{19}$ Several studies have shown that the cost-effectiveness of HCC surveillance largely depends on the incidence of HCC among at-risk population. For example, in patients with Child-Pugh A cirrhosis, surveillance for HCC becomes cost-effective if the incidence of HCC exceeds $1.5 \%$ per year. ${ }^{20}$ In patients with chronic HBV and no cirrhosis, surveillance is considered cost-effective if the incidence of HCC is greater than $0.2 \%$ per year. ${ }^{21}$ Andersson and col- leagues examined the cost-effectiveness of HCC surveillance in patients with liver cirrhosis older than 50 years old in the United States. On average, ultrasound assessment every 6 months increased quality-adjusted life expectancy among cirrhotic patients by 8.6 months. ${ }^{22}$ The results were even better in patients with small tumors where life expectancy extended to almost 3.5 years. The study observed an incremental cost-effectiveness ratio of $\$ 30,700$ per quality-adjusted life year gained. Similarly, Patel et al. demonstrated that HCC surveillance with semiannual ultrasound plus AFP in patients with compensated hepatitis $C$ virus cirrhosis gained quality-adjusted life-years by 0.49 to 3.81 at reasonable costs. ${ }^{23}$

\section{WHAT IS THE BEST SURVEILLANCE STRAT- EGY?}

Although current data suggest HCC surveillance is of value, this is dependent on having surveillance tests with sufficient sensitivity and specificity to provide an acceptable risk-benefit ratio. Currently, ultrasound is the primary modality recommended for surveillance of HCC. Although ultrasound has an acceptable sensitivity of $84 \%$ (95\% Cl 76-92\%) for detecting any-stage HCC, its sensitivity for early stage HCC detection is significantly lower at only 47\% (95\% Cl 33-61\%). ${ }^{24}$ Further, increasing data demonstrate that its effectiveness can be affected by factors including operator expertise, severity of liver disease, and patient body habitus, leading to wide variation in its sensitivity between centers and patients. ${ }^{25,26}$ There is increasing interest in alternative imaging modalities such as CT or MRI; however, these modalities are likely limited by physical harms (radiation and contrast exposure) and costs, respectively. There are ongoing studies evaluating alternative imaging strategies such as abbreviated MRI; however, until these data mature, ultrasound remains the standard radiographic surveillance modality.

Therefore, there has been increasing interest in serum biomarkers that may improve sensitivity for early tumor detection. The best studies biomarker to date is AFP, although there are few studies that directly compare the use of ultrasound alone versus ultrasound plus AFP. A recent meta-analysis found the concomitant use of AFP with ultrasound for surveillance improves detection of early-stage HCC. When AFP is used in concomitant with ultrasound, the sensitivity of detecting early-stage HCC is estimated at $63 \%,(95 \% \mathrm{Cl} 48-75 \%)$ compared to $45 \%$, $(95 \% \mathrm{Cl}$ $30-62 \%)$ for ultrasound alone. The improved sensitivity was off- 
set by a small decrease in specificity ( $84 \%$ vs. $92 \%$, risk ratio $1.08,95 \% \mathrm{Cl} 1.05-1.09)$, although the clinical significance of this decrease is likely minimal. Further, the diagnostic OR (which accounts for sensitivity and specificity) of the two tests in combination was higher than that of ultrasound alone. Finally, there are several proposals to minimize the false-positive results of AFP. First, the use of higher AFP cutoffs tends to improve specificity particularly among patients with cirrhosis secondary to viral hepatitis. ${ }^{27}$ Second, the trend of AFP test values rather than a single test result more accurately identifies patients at-risk to develop $\mathrm{HCC}^{28}$ In a recent study, a group of researchers from the VA health care system developed and validated an HCC early detection model that incorporated the rate of AFP change along with AFP most recent value, age of the patient, alanine aminotransferase blood level, and platelet count. This model was associated with improved sensitivity for early HCC detection compared to the current standard of care. ${ }^{29}$

Despite improved accuracy compared to ultrasound alone, it is clear the surveillance strategy of ultrasound and AFP remains far from ideal. The combination of two test modality still misses approximately one in three patients with HCC. A number of novel biomarkers, such as des-gamma carboxy prothrombin (DCP) and lectin-bound AFP (AFP-L3), have been promising in phase II studies $^{30}$ and currently undergoing validation in larger cohorts in phase III. Because of the heterogenicity of HCC, scientists have proposed the combination of different biomarkers rather than relying on a single biomarker in the early detecting of HCC. The GALAD panel incorporates AFP, AFP-L3, and DCP, plus patient age and gender. ${ }^{30}$ This model has shown to be promising with area under the receiver operating curve of 0.90 in case-control studies. If validated in larger studies, the GALAD model may have the potential to be used for HCC surveillance in the near future. Cell free DNA released from tumor cells, referred to as circulating tumor DNA, can be detected in peripheral blood samples and is another promising novel surveillance biomarker undergoing evaluation.

\section{HCC SURVEILLANCE UNDERUSE}

Although current data suggest HCC surveillance is of value and should be recommended to at-risk populations, several studies have shown that less than $20 \%$ of patients at risk undergo HCC surveillance. ${ }^{31}$ There have been a few intervention studies to improve utilization of HCC surveillance. For example, Beste and col- leagues demonstrated that an electronic medical reminder prompt could significantly improve HCC surveillance rates and Veterans with cirrhosis. ${ }^{32}$ Provider-directed electronic reminders improved HCC surveillance in patients with cirrhosis by $51 \%$. Similarly, Singal et al. conducted a randomized controlled study to examine the effectiveness of mailed out-reach and patient navigation strategies in order to improve surveillance of HCC in patients with liver cirrhosis. ${ }^{33}$ HCC surveillance significantly improved from $7.3 \%$ in the usual care patients to $23.3 \%$ in outreach/navigation patients. Future studies are needed to better stratify the risk for HCC and to evaluate newer surveillance strategies, including imaging and biomarkers.

\section{CONCLUSION: IS HCC SURVEILLANCE JUSTI- FIED?}

Based on current data, should we abandon HCC surveillance? Ideally, the question of whether HCC surveillance is effective would be evaluated by a randomized controlled trial of surveillance vs. no surveillance. However, such study is not feasible as HCC surveillance has largely become standard of care. When a randomized trial was attempted, most providers and patients refused to participate and wished instead of opt for surveillance. Therefore, we are forced to depend on data from cohort and modeling studies. In a recent Markov model, ${ }^{34}$ Taylor and colleagues found that surveillance was associated with $13(95 \% \mathrm{Cl}$ 12-14) fewer deaths for every 1,000 patients followed over a 5 -year period. However, they noted significant surveillance-related harms, with 150 (95\% Cl 146-154) patients having at least one false positive surveillance test, leading to 65 cross-sectional imaging (CT/MRI) studies and 39 liver biopsies. It is clear continued data are needed to evaluate both benefits and harms in representative contemporary populations to better inform assessments of surveillance value. In the interim, we believe that it is premature to abandon what has become the standard of care.

\section{Authors' contribution}

Drafting of manuscript: A. Singal and I. Hanouneh.

Critical revision and final approval: A. Singal, I. Hanouneh, and N. Alkhouri.

Study supervision: A. Singal.

\section{Funding support}

Dr. Singal's research is funded in part by NCI R01CA212008. 
The content is solely the responsibility of the authors and does not necessarily represent the official views of the National Institutes of Health.

\section{Conflicts of Interest}

Ibrahim A. Hanouneh: Speakers bureau and advisory boards for Gilead and Dova. He has served as consultant for Metavention and co-author for Skinny Liver.

Naim Alkhouri: Speakers bureau and advisory boards for Gilead and Eisai. He has served as consultant for Exelixis.

Amit G. Singal: Speakers bureau for Gilead, Bayer, and Bristol Meyers Squibb. He has served on advisory boards for Gilead, Abbvie, Bayer, Eisai, Wako Diagnostics, Roche, and Exact Sciences. He serves as a consultant to Bayer, Eisai, Roche, and Glycotest. He has received research funding from Gilead and Abbvie.

\section{REFERENCES}

1. Ferlay J, Soerjomataram I, Dikshit R, Eser S, Mathers C, Rebelo M, et al. Cancer incidence and mortality worldwide: sources, methods and major patters in GLOBOCAN 2012. Int J Cancer 2015;136:E359E386.

2. Chiang CJ, Yang YW, You SL, Lai MS, Chen CJ. Thirty-year outcomes of the national hepatitis B immunization program in Taiwan. JAMA 2013;310:974-976.

3. Petrick JL, Braunlin M, Laversanne M, Valery PC, Bray F, McGlynn $K A$. International trends in liver cancer incidence, overall and by histologic subtype, 1978-2007. Int J Cancer 2016;139:1534-1545.

4. Petrick JL, Kelly SP, Altekruse SF, McGlynn KA, Rosenberg PS. Future of hepatocellular carcinoma incidence in the United States forecast through 2030. J Clin Oncol 2016;34:1787-1794.

5. El-Serag HB. Hepatocellular carcinoma. N Engl J Med 2011;365:11181127.

6. Ascha MS, Hanouneh IA, Lopez R, Tamimi TA, Feldstein AF, Zein NN. The incidence and risk factors of hepatocellular carcinoma in patients with nonalcoholic steatohepatitis. Hepatology 2010;51:19721978.

7. Heimbach JK, Kulik LM, Finn RS, Sirlin CB, Abecassis MM, Roberts $L R$, et al. AASLD guidelines for the treatment of hepatocellular carcinoma. Hepatology 2018;67:358-380.

8. European Association for the Study of the Liver; European Organisation for Research and Treatment of Cancer. EASL-EORTC clinical practice guidelines: management of hepatocellular carcinoma. J Hepatol 2012;56:908-943.

9. Omata M, Cheng AL, Kokudo N, Kudo M, Lee JM, Jia J, et al. Asia-
Pacific clinical practice guidelines on the management of hepatocelIular carcinoma: a 2017 update. Hepatol Int 2017;11:317-370.

10. National Cancer Institute (NIH). Liver (hepatocellular) cancer screening (PDQ [R])—health professional version. NIH web site, <https:// www.cancer.gov/types/liver/hp/liver-screening-pdq>. Accessed 9 Jan 2018.

11. Rich NE, Parikh ND, Singal AG. Overdiagnosis: an understudied issue in hepatocellular carcinoma surveillance. Semin Liver Dis 2017;37:296-304.

12. Zhang BH, Yang BH, Tang ZY. Randomized controlled trial of screening for hepatocellular carcinoma. J Cancer Res Clin Oncol 2004;130:417-422.

13. Kansagara D, Papak J, Pasha AS, O’Neil M, Freeman M, Relevo R, et al. Screening for hepatocellular carcinoma in chronic liver disease: a systematic review. Ann Intern Med 2014;161:261-269.

14. Singal AG, Pillai A, Tiro J. Early detection, curative treatment, and survival rates for hepatocellular carcinoma surveillance in patients with cirrhosis: a meta-analysis. PLoS Med 2014;11:e1001624.

15. Choi DT, Kum HC, Park S, Ohsfeldt RL, Shen Y, Parikh ND, et al. Hepatocellular carcinoma screening is associated with increased survival of patients with cirrhosis. Clin Gastroenterol Hepatol 2019;17:976-987.e4.

16. Moon AM, Weiss NS, Beste LA, Su F, Ho SB, Jin GY, et al. No association between screening for hepatocellular carcinoma and reduced cancer-related mortality in patients with cirrhosis. Gastroenterology 2018;155:1128-1139.e6.

17. Atiq O, Tiro J, Yopp AC, Muffler A, Marrero JA, Parikh ND, et al. An assessment of benefits and harms of hepatocellular carcinoma surveillance in patients with cirrhosis. Hepatology 2017;65:1196-1205.

18. Konerman MA, Verma A, Zhao B, Singal AG, Lok AS, Parikh ND. Frequency and outcomes of abnormal imaging in patients with cirrhosis enrolled in a hepatocellular carcinoma surveillance program. Liver Transpl 2019;25:369-379.

19. Naimark D, Naglie G, Detsky AS. The meaning of life expectancy: what is a clinically significant gain? J Gen Intern Med 1994;9:702-707.

20. Sarasin FP, Giostra E, Hadengue A. Cost-effectiveness of screening for detection of small hepatocellular carcinoma in western patients with Child-Pugh class A cirrhosis. Am J Med 1996;101:422-434.

21. Bruix J, Sherman M; American Association for the Study of Liver Diseases. Management of hepatocellular carcinoma: an update. Hepatology 2011;53:1020-1022.

22. Andersson KL, Salomon JA, Goldie SJ, Chung RT. Cost effectiveness of alternative surveillance strategies for hepatocellular carcinoma in patients with cirrhosis. Clin Gastroenterol Hepatol 2008;6:14181424.

23. Patel D, Terrault NA, Yao FY, Bass NM, Ladabaum U. Cost-effectiveness of hepatocellular carcinoma surveillance in patients with hepatitis C virus-related cirrhosis. Clin Gastroenterol Hepatol 2005;3:75-84. 
24. Tzartzeva K, Obi J, Rich NE, Parikh ND, Marrero JA, Yopp A, et al. Surveillance imaging and alpha fetoprotein for early detection of hepatocellular carcinoma in patients with cirrhosis: a meta-analysis. Gastroenterology 2018;154:1706-1718.e1.

25. Simmons O, Fetzer DT, Yokoo T, Marrero JA, Yopp A, Kono Y, et al. Predictors of adequate ultrasound quality for hepatocellular carcinoma surveillance in patients with cirrhosis. Aliment Pharmacol Ther 2017:45:169-177.

26. Del Poggio P, Olmi S, Ciccarese F, Di Marco M, Rapaccini GL, Benvegnù $L$, et al. Factors that affect efficacy of ultrasound surveillance for early stage hepatocellular carcinoma in patients with cirrhosis. Clin Gastroenterol Hepatol 2014;12:1927-1933.e2.

27. Gopal P, Yopp AC, Waljee AK, Chiang J, Nehra M, Kandunoori P, et al. Factors that affect accuracy of a-fetoprotein test in detection of hepatocellular carcinoma in patients with cirrhosis. Clin Gastroenterol Hepatol 2014;12:870-877.

28. Lee $E$, Edward S, Singal AG, Lavieri MS, Volk M. Improving screening for hepatocellular carcinoma by incorporating data on levels of a-fetoprotein, over time. Clin Gastroenterol Hepatol 2013;11:437-440.

29. Tayob N, Christie I, Richardson P, Feng Z, White DL, Davila J, et al.
Validation of the hepatocellular carcinoma early detection screening (HES) algorithm in a cohort of veterans with cirrhosis. Clin Gastroenterol Hepatol 2019;17:1886-1893.e5.

30. Chaiteerakij R, Addissie BD, Roberts LR. Update on biomarkers of hepatocellular carcinoma. Clin Gastroenterol Hepatol 2015;13:237-245.

31. Davila JA, Morgan RO, Richardson PA, Du XL, McGlynn KA, El-Serag $H B$. Use of surveillance for hepatocellular carcinoma among patients with cirrhosis in the United States. Hepatology 2010;52:132-141.

32. Beste LA, Ioannou GN, Yang Y, Chang MF, Ross D, Dominitz JA. Improved surveillance for hepatocellular carcinoma with a primary care-oriented clinical reminder. Clin Gastroenterol Hepatol 2015;13:172-179.

33. Singal AG, Tiro JA, Murphy CC, Marrero JA, McCallister K, Fullington $\mathrm{H}$, et al. Mailed outreach invitations significantly improve HCC surveillance rates in patients with cirrhosis: a randomized clinical trial. Hepatology 2019;69:121-130.

34. Taylor EJ, Jones RL, Guthrie JA, Rowe IA. Modeling the benefits and harms of surveillance for hepatocellular carcinoma: information to support informed choices. Hepatology 2017;66:1546-1555. 